\title{
Early Results from the SMA
}

\author{
Qizhou Zhang \\ Harvard-Smithsonian Center for Astrophysics
}

\begin{abstract}
The Submillimeter Array (SMA), a collaborative project of the Smithsonian Astrophysical Observatory (SAO) and the Academia Sinica Institute of Astronomy and Astrophysics of Taiwan (ASIAA), is approaching the end of the construction phase. In this paper, I review its current capability and describe the scientific programs carried out in recent months. I will also present some preliminary results obtained with the SMA in the area of star formation.
\end{abstract}

\section{Introduction}

The Submillimeter Array project was conceived at the Smithsonian Astrophysical Observatory and proposed in 1984 to its parent organization, the Smithsonian Institute. Operating at the submillimeter window from $1.5 \mathrm{~mm}$ to 350 $\mu m$, the Array extends the capability of the existing millimeter interferometers, such as the Berkeley, Illinois and Maryland Association Array, the Owens Valley Radio Observatory Array, the IRAM Plateau de Bure Interferometer, and the Nobeyama Millimeter Array. In 1992, funding was committed to build an array of six 6-meter dishes. In 1996, the Institute of Astronomy and Astrophysics of Academic Sinica in Taiwan joined the collaboration to build two additional dishes, expanding the array to 8 elements.

Various sites including western United States, northern Chile and Mauna Kea, Hawaii were considered for the SMA. A joint agreement between the SAO and the Institute for Astronomy, University of Hawaii was reached in 1994, allowing the SMA to locate and operate in the submillimeter valley at the summit of Mauna Kea, Hawaii. A recent picture of the SMA is shown in Figure 1.

\section{The Basics of the SMA and Current Status}

The SMA, when completed, consists of 8 parabolic reflectors with a diameter of 6 meters. Antennas can be arranged in 4 different array configurations, each of which form an approximate shape of a Reuleaux triangle. This antenna configuration gives the optimum UV coverage with a circular boundary (Keto 1997). The baselines between the configurations scale up by a factor of 2.7 . The most sparse antenna configuration has a maximum baseline of $508 \mathrm{~m}$ and a synthesize beam of $0^{\prime \prime} .1$ at $820 \mathrm{GHz}$, the highest frequency band for the SMA. The cryogenically cooled double sideband receivers operate in five frequency bands around $230 \mathrm{GHz}, 345 \mathrm{GHz}, 460 \mathrm{GHz}, 690 \mathrm{GHz}$ and $820 \mathrm{GHz}$. Two 


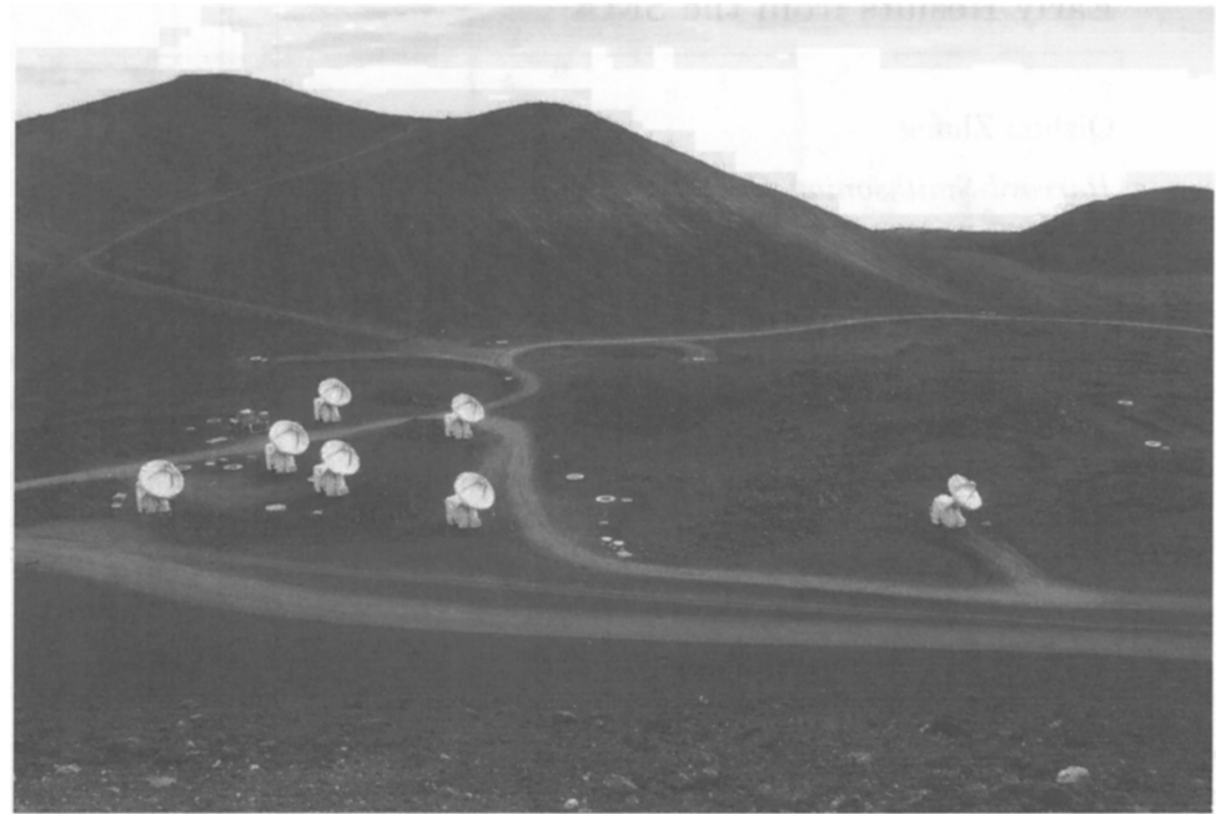

Figure 1. A recent picture of the SMA at Mauna Kea, Hawaii showing seven antennas.

receivers can operate simultaneously, allowing for observations at two different frequency bands, or at the same frequency to enhance sensitivity or to study polarization.

The correlator processes signals from dual sidebands separated by $10 \mathrm{GHz}$. Each sideband provides a bandwidth of $2 \mathrm{GHz}$ at a default spectral resolution of $0.8 \mathrm{MHz}$ across the band. The broad bandwidth combined with fine spectral resolution provides a unique capability studying the broad line emission. The high spectral resolution $(0.025 \mathrm{MHz} / \mathrm{Channel})$ mode will be implemented, allowing the study of narrow spectral lines from regions such as quiescent molecular cloud cores.

In the past few months, the array has been operating routinely in the interferometry mode with 4-6 antennas and a bandwidth of $1 \mathrm{GHz}$. Most of the operations were at 230 and $345 \mathrm{GHz}$ bands. Some testings at the $690 \mathrm{GHz}$ band were also carried out. Phase closure and imaging with three antennas have been achieved at $690 \mathrm{GHz}$. Table 1 gives a summary of the SMA specification and current status. 
Table 1. SMA Specification and Current Status (as of 2003/08/30)

\begin{tabular}{lll}
\hline \hline & Goal & Current \\
\hline Antennas & & \\
6m dishes & 8 & $15-20 \mathrm{~m} \mathrm{rms}$ \\
Surface accuracy & $12 \mathrm{~m} \mathrm{rms}$ & \\
Aperture efficiency & & $0.70-0.80$ \\
$230 \mathrm{GHz}$ & 0.79 & $0.55-0.65$ \\
$345 \mathrm{GHz}$ & 0.77 & 0.40 (preliminary) \\
$690 \mathrm{GHz}$ & 0.70 & $4^{\circ} / \mathrm{s}$ \\
Slew rate & $4^{\circ} / \mathrm{s}$ & \\
\hline Configuration & & $16-120 \mathrm{~m}$ \\
Baselines & $8-508 \mathrm{~m}$ & $1^{\prime \prime}$ \\
Resolution $(345 \mathrm{GHz})$ & $0^{\prime \prime} .2$ & $70^{\prime \prime}-14^{\prime \prime}$ \\
Field of View & $70^{\prime \prime}-14^{\prime \prime}$ & \\
\hline Receivers & & 7 \\
$230 \mathrm{GHz}$ & 8 & 7 \\
$345 \mathrm{GHz}$ & $8 \times 2 \mathrm{polarizations}$ & 0 \\
$460 \mathrm{GHz}$ & 8 & 4 \\
$690 \mathrm{GHz}$ & 8 & 0 \\
$820 \mathrm{GHz}$ & 8 & $1 \mathrm{GHz}$ \\
Bandwidth & $2 \mathrm{GHz}$ & $0.2 \mathrm{MHz}$ \\
Resolution & $0.025 \mathrm{MHz}$ & \\
\hline Sensitivity (6 hours) & & \\
$230 \mathrm{GHz}$ & $0.4 \mathrm{mJy}$ & \\
$345 \mathrm{GHz}$ & $1.0 \mathrm{mJy}$ & \\
$690 \mathrm{GHz}$ & $10 \mathrm{mJy}$ & \\
\hline Data Format & & \\
\hline \hline
\end{tabular}

${ }^{1}$ Data are transferable to FITS and MIRIAD format.

\section{Preliminary Results from the SMA}

As the first imaging interferometer at the submillimeter wavelengths, SMA is a powerful tool for studying a wide range of astrophysical problems, such as the dusty regions in molecular clouds, around stars and in galaxies. Despite the small collecting area $\left(226 \mathrm{~m}^{2}\right)$ as compared to other millimeter-wave interferometers, it provides a better sensitivity in brightness temperature to dust emission due to the $\nu^{4}$ dependence of the flux from the dust emission. The broad bandwidth of the SMA allows efficient observations of abundant molecular line transitions from high $\mathrm{J}$ energy levels. Figure 2 shows lines of many hot-core type molecules toward a high-mass (proto)star IRAS 18089-1732 (Beuther et al. in preparation). About 20 emission lines can be mapped simultaneously in one sideband. The multiple lines allow us to investigate the physical conditions, the kinematics and chemistry of this high-mass star forming region (See Beuther's contribution 


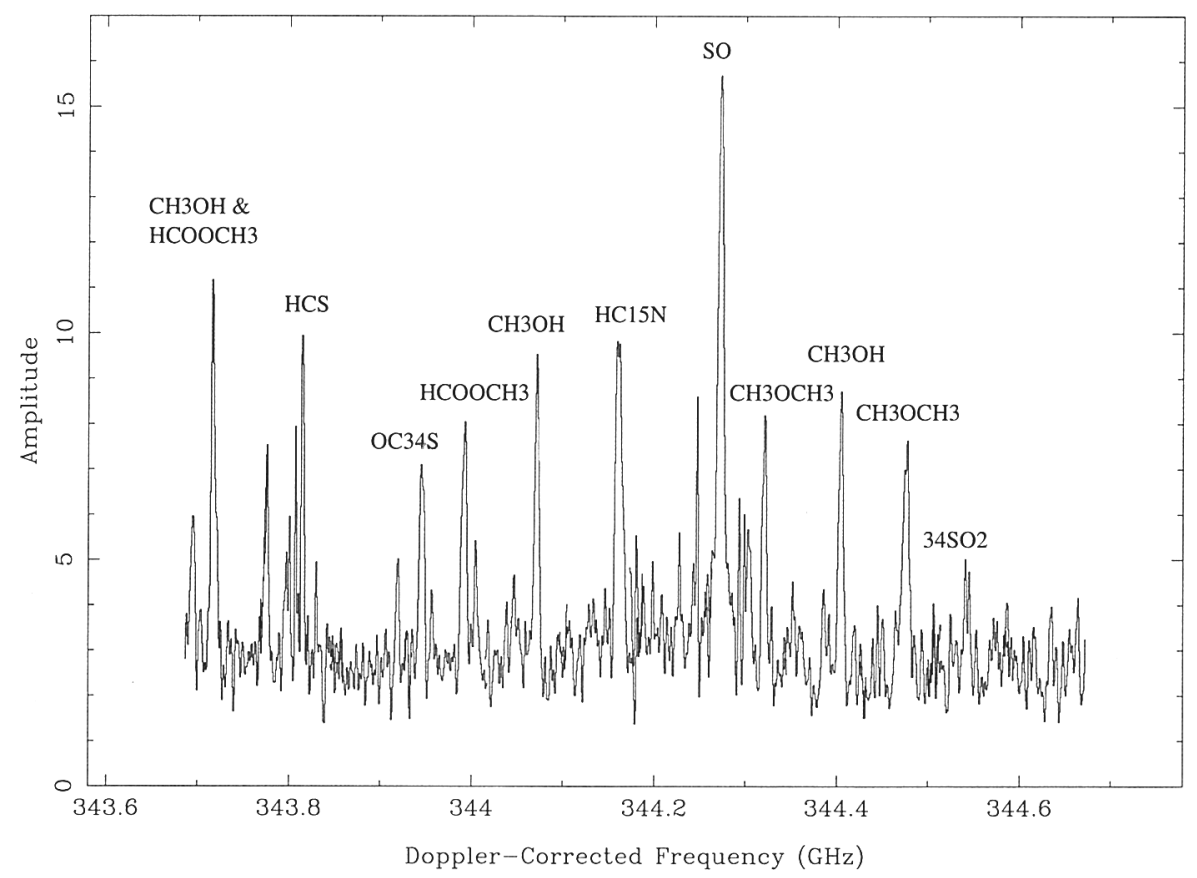

Figure 2. The visibility amplitude across the $1 \mathrm{GHz}$ band for IRAS $18089-1732$.

in this proceedings). The fine spectral resolution across the broad frequency band makes it easier to image broad spectral lines from galaxies and the pressure broadened lines from planet's atmosphere. The submillimeter window also provides a unique probe of the non-thermal emission from the black hole toward the Galactic Center, since the emission from the shorter wavelengths is thought to arise from the inner regions of the accretion disk (Zhao et al. 2003).

In the past few years, scientific programs were carried out along with major engineering efforts during the construction and commissioning phase of the array. In 2002, projects in seven scientific categories were established. The studies consist of solar system objects, nearby star forming regions and disks, massive star forming regions, the Galactic Center, nearby galaxies, and distant galaxies. Most of the observations were carried out at 230 and $345 \mathrm{GHz}$ with 4 to 6 antennas. For objects with southern declinations, additional observations were done in a more sparse configuration with one antenna relocated to provide better angular resolution in the north-south direction.

Limited observations were also carried out at the $690 \mathrm{GHz}$ band with 3 or 4 antennas. In particular, first interferometric images at the $690 \mathrm{GHz}$ band were made toward the carbon star IRC $+10^{\circ} 216$. The observations resolve the $\mathrm{CS}$ 
$\mathrm{J}=14-13$ emission in the envelope and allow the study of chemistry in the region (Young et al. in preparation).

Many of the SMA early scientific results will be submitted for publication in the next few months. Here, I present some preliminary images. Because of the limited space, I will focus on few science projects germane to star formation. Additional results from the SMA can be found in contributions in this proceedings by Beuther (IRAS 18089-1732), Ohashi (L1551) and Wilner (TW Hya).

\subsection{G5.89-0.39: A massive star forming region}

G5.89-0.39 is an ultra compact HII (UCHII) region at a distance of 3 to 7 kpc. Its high infrared luminosity of $3 \times 10^{5} \mathrm{~L}_{\odot}$ indicates an equivalent zeroage-main-sequence star of O6.5. The UCHII region is known to expand based on comparison of $\mathrm{cm}$ continuum emission from different epochs (Kawamura \& Masson 1996; Acord, Churchwell \& Wood 1998). One of the peculiar aspects of G5.89 is the extremely high velocity gas toward the object: CO emission has broad wings upto $140 \mathrm{~km} \mathrm{~s}^{-1}$ in full width (Choi, Evans, Jaffe 1993). Maps in CO 2-1 and 3-2 from the single dish telescope show a spatially unresolved outflow (Choi et al. 1993). High velocity $\mathrm{SiO}$ gas has also been observed in the region (Acord, Walmsley \& Churchwell 1997).

SMA obtained images in the $\mathrm{SiO} J=5-4$ line and $217 \mathrm{GHz}$ continuum (Sollins et al. in preparation). As seen in Figure 3, a spatially well resolved $\mathrm{SiO}$ outflow is apparent. The $\mathrm{SiO}$ line wings extends to more than $80 \mathrm{~km} \mathrm{~s}^{-1}$.

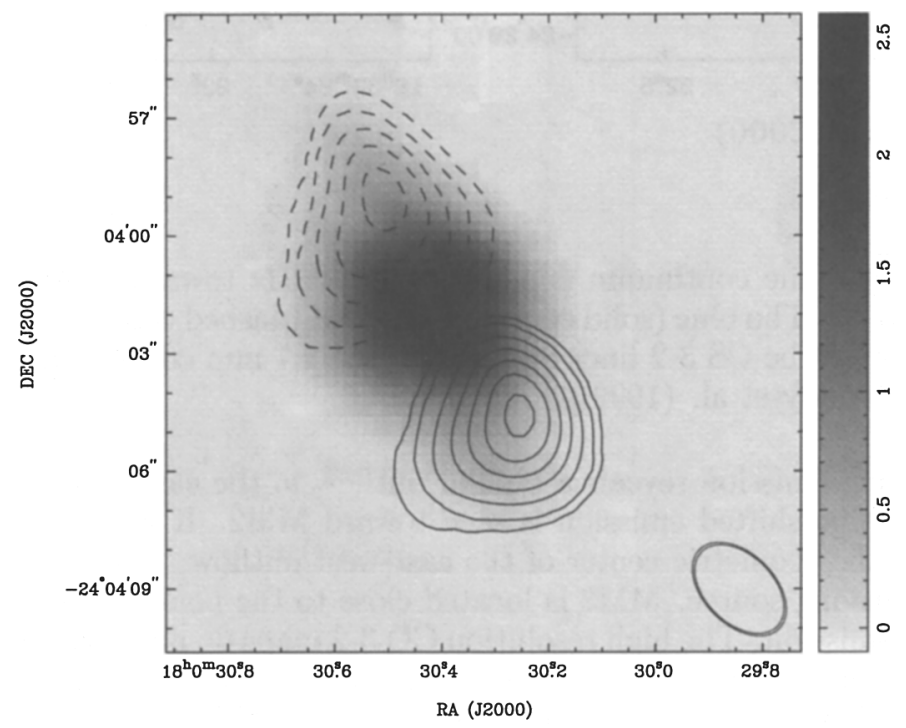

Figure 3. G5.89-0.38: $\mathrm{SiO}$ emission in the outflow overlaid on the $217 \mathrm{GHz}$ continuum. The solid contours are the blue-shifted lobe. The dashed contours are the red-shifted lobe. The continuum emission is in grey scales. 


\subsection{IRAS 16293-2422}

IRAS 16293-2422 is a well known proto binary system located in the east of the $\rho$ Ophiuchi cloud complex at a distance of 160 pc. Mizuno et al. (1990) reported a complex quadrupolar $\mathrm{CO}$ outflow. At mm continuum, two dust peaks are detected at a projected distance of 840 AU (Mundy et al. 1992; Looney et al. 2000).

Figure 4 presents images toward IRAS $16293-2422$ at the $349 \mathrm{GHz}$ band (Takakuwa, Hirano, private communication). With $<2^{\prime \prime}$ angular resolution, the SMA resolves the two dust continuum peaks. The positions are in general agreement with these measured at $2.7 \mathrm{~mm}$. The continuum peak toward the southeast (MM1) is resolved.

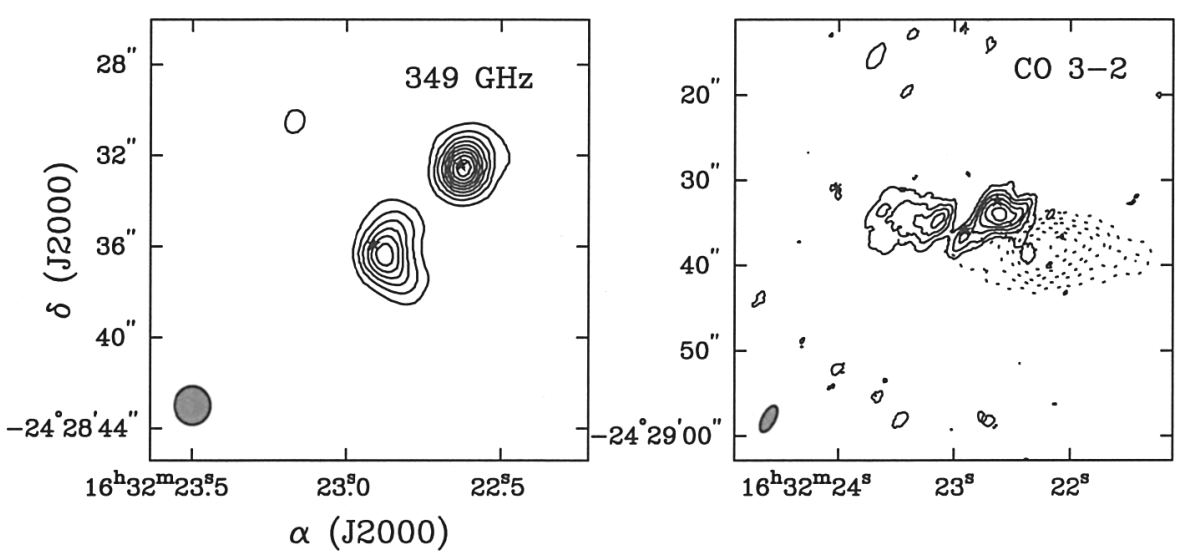

Figure 4. Left: The continuum emission at $349 \mathrm{GHz}$ toward IRAS 16293-2422. Right: The blue (solid contours) and red (dashed contours) shifted emission in the CS 3-2 line. Stars mark the $2.7 \mathrm{~mm}$ continuum position from Mundy et al. (1992).

The CO 3-2 wing emission reveals a bipolar outflow in the east-west orientation. Additional blue shifted emission is seen toward MM2. It appears that MM1 is located at the geometric center of the east-west outflow. It is a strong candidate for the driving source. MM2 is located close to the peak of the additional blue-shifted emission. The high resolution CO 3-2 maps do not correspond well with the single dish map in the CO 1-0 by Mizuno et al. (1990). In particular, the counterpart of the second pair of lobes along the NE-SW direction is not clear in the interferometric maps.

\subsection{LkCa 15}

LkCA 15, located in the Taurus cloud complex at a distance of $140 \mathrm{pc}$, is a classical $\mathrm{T}$ Tauri star with a large gaseous disk. High resolution images were 
obtained in the CO 2-1 line, revealing Keplerian motions in the disk (Duvert et al. 2000).

SMA obtained images in continuum at $345 \mathrm{GHz}$ and the $\mathrm{CO} 3-2$ line (see Figure 5) (Qi, private communication). The $\mathrm{CO}$ emission is resolved and a velocity gradient characteristic of Keplerian motion is detected.
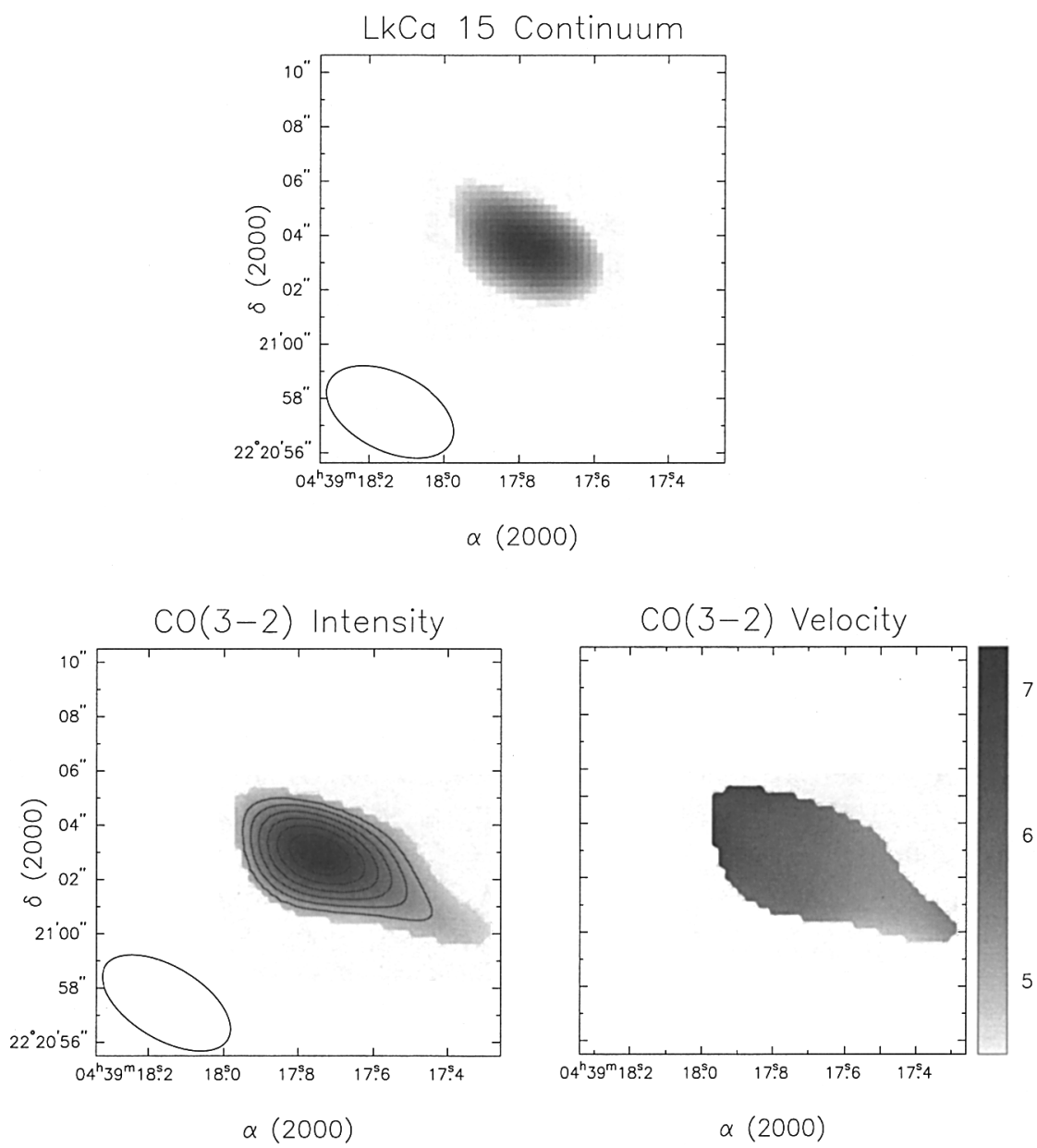

Figure 5. Top: Continuum emission at $345 \mathrm{GHz}$. Bottom: The integrated emission and the centroid velocity of the CO 3-2 transition. The ellipse at the lower-left corner of each panel is the size of the synthesized beam. 


\section{Development in the Next Few Months}

Toward the end of year 2003, the SMA is expected to operate with 8 antennas and the full $2 \mathrm{GHz}$ bandwidth. Within the next two years, another set of receivers covering the $345 \mathrm{GHz}$ band will be developed. The dual band operation will improve the sensitivity or allow polarization studies at $345 \mathrm{GHz}$. The James Clerk Maxwell Submillmeter Telescope (JCMT) and the Caltech Submillimeter Observatory (CSO) telescope will be linked with SMA to provide better point source sensitivity and spatial resolution. We look forward to a fruitful scientific endeavor of the SMA in years to come.

For up-to-date information on the SMA, visit http://sma-www . harvard.edu.

Acknowledgments: The SMA involves the team effort of dedicated staff from both SAO and ASIAA. QZ thanks H. Beuther, M. Gurwell, N. Hirano, D. Iono, A. Peck, P. Sollins, C. Qi, K. Sakamoto, H. Shinnaga, S. Takakuwa, J. Wang, D. Wilner and J. Zhao for providing images prior to publication. QZ is grateful to H. Beuther, N. Hirano and S. Takakuwa for comments on the manuscript.

\section{References}

Acord, J. M., Churchwell, E. \& Wood, D. O. S. 1998, ApJ, 495, L107

Acord, J. M., Walmsley, C. M., \& Churchwell, E. 1997, 475, 693

Choi, M., Evans, N. J. II, Jaffe, D. 1993, ApJ, 417, 624

Duvert, G., Guilloteau, S., Ménard, F., \& Dutrey, A. 2002, A\&A, 355, 165

Kawamura, J., \& Masson, C. R. 1996, ApJ, 461, 282

Keto, E., 1997, ApJ, 475, 843

Looney, L, Mundy, L. G., \& Welch, W. J. 2000, ApJ, 529, 477

Mizuno, A., Fukui, Y., Iwata, T., \& Nozawa, S. 1990, ApJ, 356, 184

Moran, J. M. 1998, in Proc. SPIE Vol3357, the Submillimeter Array, ed. T. G. Phillips, 208

Mundy, L. G., Wootten, A., Wilking, B. A., et al. 1992, ApJ, 385, 306

Zhao, J-H., Young, K. H., Herrnstein, R. M., et al. 2003, ApJ, 586, L29 


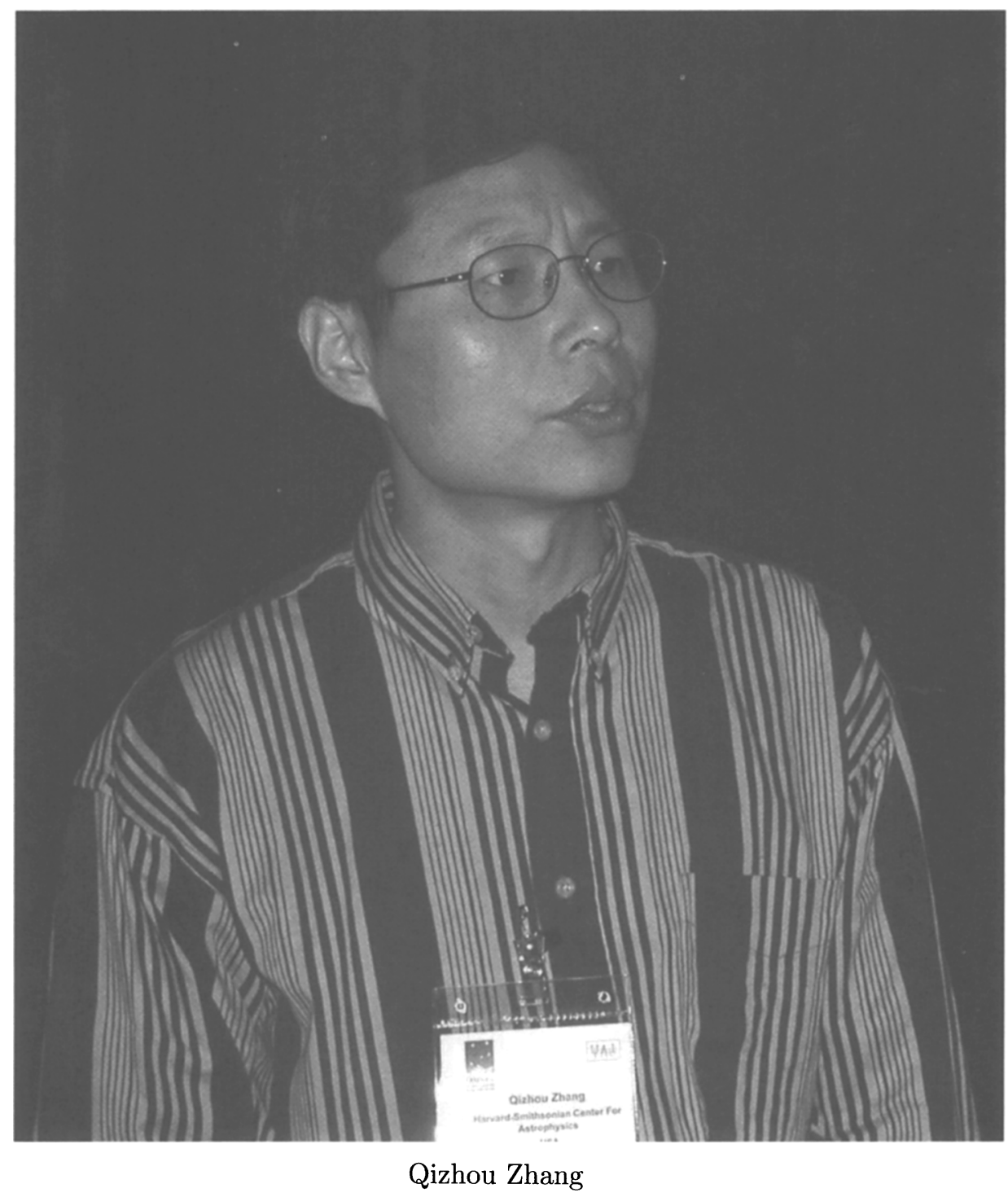




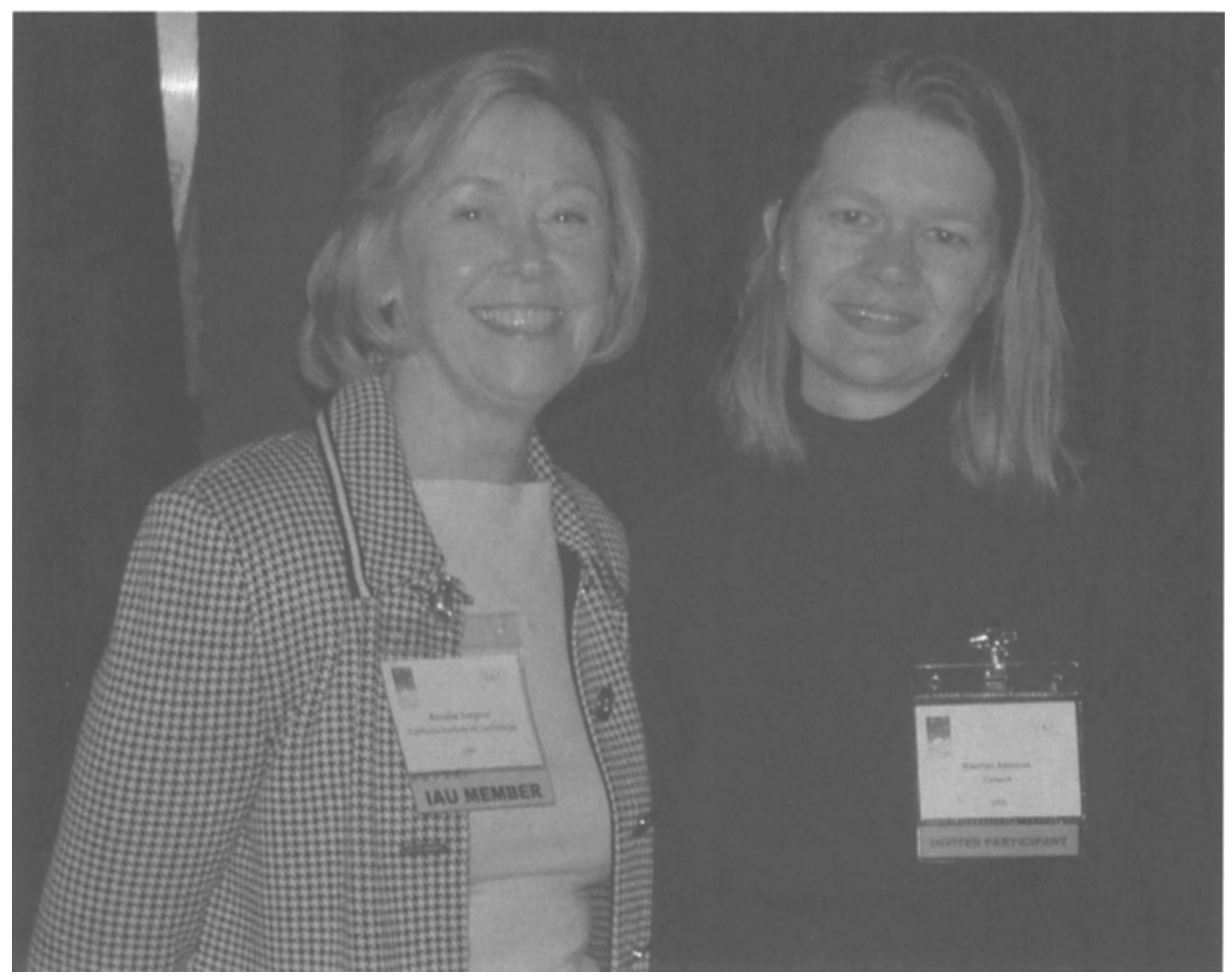

Anneila Sargent and Rachel Akeson 